\title{
Effects of Active Vibration Exercise on Neck Pain, Disability Index, and Muscle Activity of Patients with Forward Head Posture
}

\author{
Yong Nam Kim¹, Dong Kyu Lee² \\ 'Department of Physical Therapy, Nambu University, Gwangju; ²Department of Physical Therapy, Sunhan Hospital, Gwangju, Korea
}

Purpose: The purpose of this study was to evaluate the impact of active vibration exercise on the neck pain, disability index, and muscle activity of patients with forward head posture.

Methods: A total of 24 patients were randomly assigned to an experimental group or a control group $(\mathrm{n}=12$ each). The experimental group performed active vibration exercise using a flexi-bar for 20 minutes a day, five times a week for four weeks. The study measured patient neck pain using a visual analog scale, neck pain related disability using the neck disability index, and muscle activity using electromyography.

Results: The intragroup comparison showed significant differences in the visual analog scale score, neck disability index score and upper trapezius, lower trapezius and serratus anterior muscle activity values among patients in the experimental group. The intergroup comparison showed that differences in the visual analog scale score, neck disability index score and upper trapezius, lower trapezius and serratus anterior muscle activity values in the control group.

Conclusion: This study showed that active vibration exercise was effective in improving the neck pain, disability index, and muscle activity of patients with forward head posture.

Keywords: Vibration, Pain, Muscle activity

\section{INTRODUCTION}

Neck pain is a musculoskeletal system disease that may be experienced more than once during a lifetime and causes headaches, reduced joint range of motion, and functional limitations. ${ }^{1,2}$ It may develop into neck pain-related disability. ${ }^{3}$ Disability related to chronic neck pain has increased in recent years..$^{1,3}$ One such disability is forward head posture which leads to the hyperlordosis of upper cervical vertebra, protraction of the jaw, and continuous loading on the joints and muscles, causing fatigue and pain. ${ }^{3}$ Wrong postures such as forward head posture can cause abnormal distortion of the spine leading to a disease accompanied by stiffening and pain. ${ }^{3,4}$ In addition, habitual forward head posture exacerbates the deep neck flexor muscle, which causes neck pain and abnormal curvature. ${ }^{4}$ The forward head posture exacerbates the muscles around shoulder bones such as lower trapezius and wide latissimus dorsi, causes ex-

Received Nov 13, 2018 Revised Dec 21, 2018

Accepted Dec 26, 2018

Corresponding author Dong Kyu Lee

E-mail Idkpt@hanmail.net cessive tension, changes the length of scalene muscles, and the muscle levator scapulae, sternocleidomastoid muscle, and upper trapezius to support the weight of protruded head. ${ }^{3-5}$ Decker et al. ${ }^{5}$ said that it is necessary to increase the muscle activity of serratus anterior, the stabilizer muscles of shoulder joint, and the middle trapezius and lower trapezius while decreasing the muscle activity of upper trapezius. To improve neck pain and forward head posture, electric treatment, therapeutic exercise, joint mobilization exercise, and manipulation are used. ${ }^{6}$ While current treatment methods consist primarily of passive approaches carried out by physical therapists, vibration exercise using flexi-bar is considered an active exercise method.

Active vibration exercise creates hypergravity by causing vibration frequency and high acceleration of amplitude and induces repeated concentric and eccentric contraction on muscles. ${ }^{7.8}$ Such state stimulates muscle spindle and activates proprioceptor and golgi tendon organ, and excitability input information by vibration

Copylight (C)2018 The Korea Society of Physical Therapy

This is an Open Access article distribute under the terms of the Creative Commons Attribution Non-commercial License (Http:// creativecommons.org/license/by-nc/4.o.) which permits unrestricted non-commercial use, distribution, and reproduction in any medium, provided the original work is properly cited. 
stimulation receives the concentric nerve fiber stimulation of I and II and delivers it to the spinal cord through the polysynatic route of spinal cord ${ }^{8,9}$ After connecting with the a-exercise nerve located inside the spinal cord, the information is sent to the extrafugal muscle fiber of agonistic muscle through are muscle movement, increasing muscle creation. ${ }^{7,9}$ The mechanical stimulation occurred through this route causes tonic vibration reflex which brings short and fast changes to the length of muscle-tendon complex, induces the contraction and relaxation of muscles, and activates neuromuscular system..$^{7-9}$ Especially, active vibration exercise is used with a flexi-bar for the physical functional recovery and therapy of patients with musculoskeletal system. ${ }^{10}$ Lee et al. ${ }^{11}$ reported that active vibration exercise using a shoulder joint is effective for relieving the pain and functional disability of chronic backache patients and Mileva et al. ${ }^{10}$ also reported that active vibration exercise using flexi-bar has a positive impact on improving muscle activities. An active vibration exercise using flexi-bar is effective for muscle coordination because it causes tonic vibration reflex in enthesis and stimulates the proprioceptive senses of joint, and because the vibration leads to contraction in turns on agonist and antagonist to adjust instability during an exercise. ${ }^{10-12}$ Vibration exercise using a flexi-bar is reportedly effective at improving trunk muscle activity and balance. ${ }^{13}$

However, there is limited research currently available on the effects of active vibration exercise on patients with forward head posture to improve their pain, disability index, and muscle activity. Thus, the purpose of this study was to evaluate the effects of active vibration exercise on the pain and disability index, and muscle activity of patients with forward head posture.

\section{METHODS}

\section{Subjects}

This study enrolled 24 patients with forward head posture with a craniovertebral angle (CVA) less than $52^{\circ}$, and randomly assigned them to either the experimental group or the control group with 12 participants (6 men, 6 women) in each group. The research participants selected received a thorough explanation related to the research objectives and methods before the experiment and voluntarily agreed to participate. This study complied with the ethical standards of the declaration of Helsinki. Patients with a previous diagnosis of a musculoskeletal system disease over the last 6 months, severely unstable spine, and osteoporosis, and vascular disease were excluded from the study. The general characteristics of research subjects are shown in Table 1.

\section{Interventions}

The experimental and control groups received general physical therapy. General physical therapy was carried out in 3 ways including 20 minutes for superficial heat therapy, 5 minutes for deep heat therapy, and 20 minutes for electric therapy, all 5 times a week for 4 weeks. In addition, the experimental group performed an active vibration workout for 20 minutes a day, five times a week, for 4 weeks using a flexi-bar (FLEXI-BAR ${ }^{\circledR}$; Flexi-Sports, Germany). The flexibar is a stick that is $1,520 \mathrm{~mm}$ long and weighs $719 \mathrm{~g}$. The middle part of the stick has a rubber handle measuring $17.9 \mathrm{~cm}$ long, whereas the ends consist of weighted rubber, allowing the hands and arms to transfer approximately $5 \mathrm{~Hz}$ vibrations when holding the middle handle and shaking the stick. The flexi-bar is vibrating

Table 1. General characteristics of study subjects

\begin{tabular}{lccc}
\hline & $E G(n=12)$ & $C G(n=12)$ & $p$ \\
\hline Sex (male/female) & $6 / 6$ & $6 / 6$ & \\
Age (year) & $49.3 \pm 1.5$ & $51.3 \pm 2.5$ & 0.317 \\
Height $(\mathrm{cm})$ & $163.3 \pm 2.3$ & $160.3 \pm 2.9$ & 0.233 \\
Weight $(\mathrm{kg})$ & $66.3 \pm 5.4$ & $67.7 \pm 4.9$ & 0.774 \\
CVA $\left({ }^{\circ}\right)$ & $45.4 \pm 0.6$ & $43.7 \pm 1.2$ & 0.089
\end{tabular}

Values are presented as mean \pm standard deviation.

EG: experimental group, CG: control group, CVA: craniovertebral angle.

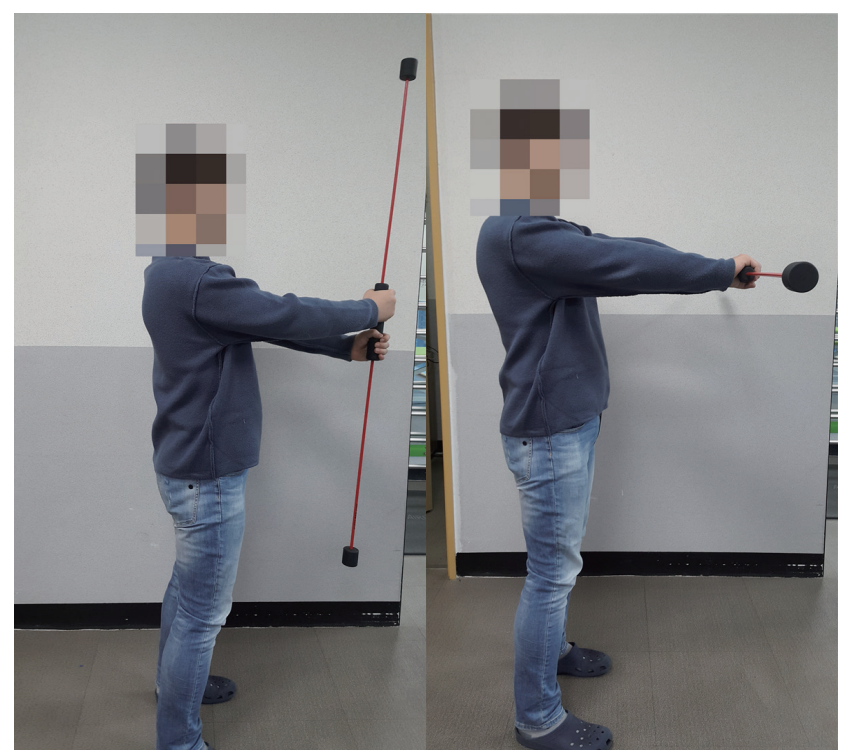

Figure 1. An active vibration exercise using flexi-bar. 
exercise equipment that uses $4.6 \mathrm{~Hz}$ vibrations generated by shaking the $153 \mathrm{~cm}$-long stick, which activates muscle spindles and helps strengthen the muscles involved in the proprioceptive sense and posture stability by providing a strong sensory stimulus. ${ }^{10,11}$ Vibration exercise using the flexi-bar is reportedly effective for nerve control ability by causing the co-contraction of targeted muscle groups. ${ }^{10,12}$ Subjects held the Flexi-Bar with both hands while standing and performed an up-and-down exercise motion to generate vibrations (Figure 1).

\section{Measurements}

Patient visual analog scale (VAS), neck disability index (NDI) score, and muscle activity were recorded before and after the 4 weeks of treatment. The VAS was used to measure pain severity. A linear scale marked 0-10 shows the extent of pain. No pain is defined as 0 , maximum pain is 10. VAS is also a highly reliable evaluation method that had study subjects self-report degree of pain.

The extent of disability caused by neck pain was measured by the NDI. The NDI is a 6 point scale consisting of 10 questions examining pain, daily life, lifting objects, reading, headache, concentration, sleep, driving, work, and leisure for which patients self-report answers. A higher score was defined as a more severe functional disability. Scores were divided into 0-4 points for no disability, 5-14 points mild disability, 15-24 points moderate disability, 25-34 points severe disability, and 35 or higher for complete disability.

Muscle activity was measured using surface electromyography (LXM 5,308, Laxtha Inc., Daejeon, Korea). The configuration was set at a sampling rate of $1,024 \mathrm{~Hz}$, band pass filter of $20-450 \mathrm{~Hz}$, and notch filter of $60 \mathrm{~Hz}$. The obtained muscle activity signals were analyzed using electromyogram software (Telescan 3.11, Laxtha Inc., Daejeon, Korea), by processing with root mean square (RMS). Body parts with attached surface electrodes were rubbed with sandpaper, and the outermost layer of the skin was removed with cotton swabs containing alcohol to reduce skin resistance. The surface electrodes were attached to the upper upper trapezius, lower trapezius and serratus anterior muscles. For normalization of the surface electromyogram signal, reference voluntary contraction was used. Electromyogram signal was used to measure the patients' posture. To evaluate posture, participants stood up with their chin tucked in, fully unfolded their elbow joint, had both arms facing outward while having their palm facing upward, and held $1 \mathrm{~kg}$-dumbbells in both hands for 5 seconds. Movements were measured three times, and the average value was obtained to minimize measurement errors; the measurement time was analyzed by collecting 3-second signals that eliminated the first and last 1 second and applying \% RVC value from the electromyogram signal recorded by measuring for 5 second.

\section{Statistical analysis}

Collected data were analyzed using SPSS 19.0. Descriptive statistics were used to compare the general characteristics of the participants. All data was verified for normality through the Shapiro-Wilks verification test. The paired t-test was used to compare values before and after the experiment. An independent t-test was conducted to compare intergroup differences among changes before and after the experiment. The statistical significance level was set at $\alpha=0.05$.

\section{RESULTS}

\section{Visual analog scale}

The experimental group had a significant difference as its VAS score decreased from $8.80 \pm 1.64$ to $4.80 \pm 0.83(\mathrm{p}<0.05)$, but the control group did not have a significant difference even though its VAS score decreased from $7.20 \pm 2.48$ to $5.20 \pm 2.77(\mathrm{p}>0.05)$ (Table 2). The experimental group had more significant difference than the control group when the groups were compared before and after the experiment $(\mathrm{p}<0.05)$ (Table 3$)$.

\section{Neck disability index}

The experimental group had a significant difference as its neck dis-

Table 2. The comparison of variable on pre and post in inner-group

\begin{tabular}{lccccl}
\hline & Group & Pre & Post & $\mathrm{t}$ & \multicolumn{1}{c}{$\mathrm{p}$} \\
\hline VAS (score) & EG & $7.96 \pm 1.34$ & $3.86 \pm 1.09$ & 2.841 & $0.001^{*}$ \\
& CG & $6.96 \pm 0.90$ & $5.33 \pm 0.57$ & 1.994 & 0.184 \\
NDI (score) & EG & $8.97 \pm 0.32$ & $2.43 \pm 0.15$ & 2.217 & $0.002^{*}$ \\
& CG & $7.66 \pm 2.08$ & $5.93 \pm 0.55$ & 1.143 & 0.372 \\
UT (\%RVC) & EG & $53.48 \pm 1.26$ & $42.38 \pm 2.55$ & 6.474 & $0.023^{*}$ \\
& CG & $55.71 \pm 3.89$ & $53.28 \pm 1.19$ & 1.084 & 0.392 \\
LT (\%RVC) & EG & $42.05 \pm 1.65$ & $49.38 \pm 0.90$ & -1.938 & $0.008^{*}$ \\
& CG & $38.92 \pm 0.70$ & $39.21 \pm 1.71$ & -0.219 & 0.847 \\
SA (\%RVC) & EG & $39.16 \pm 2.82$ & $50.96 \pm 3.42$ & -4.996 & $0.001^{*}$ \\
& CG & $39.04 \pm 4.73$ & $41.10 \pm 3.97$ & -0.698 & 0.558 \\
\hline
\end{tabular}

Values are presented as mean \pm standard deviation, ${ }^{*} p<0.05$.

EG: experimental group, CG: control group, VAS: visual analog scale, NDI: neck disability index, UT: upper trapezius, LT: lower trapezius, SA: serratus anterior. 
Table 3. The comparison of changes in variables between groups

\begin{tabular}{lrccc}
\hline & \multicolumn{1}{c}{ EG } & CG & $\mathrm{t}$ & $\mathrm{p}$ \\
\hline VAS (score) & $-4.10 \pm 0.26$ & $-1.63 \pm 1.41$ & -2.960 & $0.042^{*}$ \\
NDI (score) & $-6.54 \pm 0.46$ & $-1.73 \pm 2.62$ & -3.120 & $0.036^{*}$ \\
UT (\%RVC) & $-11.05 \pm 2.95$ & $-2.42 \pm 3.88$ & -3.062 & $0.038^{*}$ \\
LT (\%RVC) & $7.32 \pm 1.15$ & $0.29 \pm 2.30$ & 4.717 & $0.009^{*}$ \\
SA (\%RVC) & $14.72 \pm 0.60$ & $2.05 \pm 5.10$ & 4.282 & $0.013^{*}$ \\
\hline
\end{tabular}

Values are presented as mean \pm standard deviation, ${ }^{*} \mathrm{p}<0.05$.

EG: experimental group, CG: control group, VAS: visual analog scale, NDI: neck disability index, UT: upper trapezius, LT: lower trapezius, SA: serratus anterior.

ability index score decreased from $8.97 \pm 0.32$ to $2.43 \pm 0.15(\mathrm{p}<0.05)$, but the control group did not have a significant difference even though its neck disability index score decreased from $7.66 \pm 2.08$ to $5.93 \pm 0.55$ ( $p>0.05$ )(Table 2). The experimental group had more significant difference than the control group when the groups were compared before and after the experiment $(\mathrm{p}<0.05)$ (Table 3).

\section{Muscle activity}

\section{1) Upper trapezius muscle}

The experimental group had a significant difference as its upper trapezius muscle decreased from $53.48 \pm 1.26$ to $42.38 \pm 2.55$ ( $\mathrm{p}<$ $0.05)$, but the control group did not have a significant difference even though its neck disability index score decreased from $55.71 \pm$ 3.89 to $53.28 \pm 1.19$ ( $>0.05$ )(Table 2). The experimental group had more significant difference than the control group when the groups were compared before and after the experiment $(\mathrm{p}<0.05)$ (Table 3 ).

\section{2) Lower trapezius muscle}

The experimental group had a significant difference as its lower trapezius muscle increased from $42.05 \pm 1.65$ to $49.38 \pm 0.90$ ( $p<0.05)$, but the control group did not have a significant difference even though its lower trapezius muscle increased from $38.92 \pm 0.70$ to $39.21 \pm 1.71$ ( $p>0.05$ )(Table 2). The experimental group had more significant difference than the control group when the groups were compared before and after the experiment $(\mathrm{p}<0.05)$ (Table 3$)$.

\section{3) Serratus anterior muscle}

The experimental group had a significant difference as its serratus anterior muscle increased from $39.16 \pm 2.82$ to $50.96 \pm 3.42(\mathrm{p}<0.05)$, but the control group did not have a significant difference even though its serratus anterior muscle increased from $39.04 \pm 4.73$ to $41.10 \pm 3.97$ ( $p>0.05$ )(Table 2). The experimental group had more significant difference than the control group when the groups were compared before and after the experiment $(\mathrm{p}<0.05)$ (Table 3$)$.

\section{DISCUSSION}

This study aimed to identify the effects of active vibration exercise on the pain, disability, and muscle activity of patients with forward head posture. There was no significant difference among the pain, $\mathrm{NDI}$, and muscle activity in the control group that was given the general physical therapy, which is considered helpful for simple pain adjustment. Pain decreased after the use of active vibration exercise in patients with forward head posture. Previously, Park et al. ${ }^{14}$ reported that active vibration exercise using body blade had a positive impact on pain relief, and Lee et al. ${ }^{11}$ additionally reported that active vibration exercise using the shoulder joint was effective for pain relief, supporting our study results. Vibration exercise is helpful for pain relief by stimulating the proprioceptive sense and activating nerve fibers. ${ }^{15}$ Active vibration exercise using flexi-bar generates 4.5 $\mathrm{Hz}$ of strong vibration stimulus, reducing pain by inducing the relative activation of $A \beta$ nerve fiber which is a low threshold mechanical receptor. ${ }^{10,12}$ It is considered that an active vibration exercise using flexi-bar relaxed muscles around the neck, which decreased the neck pain of patients with the forward head posture.

Kim et al. ${ }^{16}$ reported a decrease in the NDI score after applying active vibration exercise to patients with forward head posture, and Lee et al. ${ }^{11}$ reported that active vibration exercise using shoulder joint decreased the functional disability index of patients. Since the active vibration exercise using flexi-bar in this study improved both neck functions and the ability to maintain posture, it is possible that the NDI decreased because of reduced stress on muscles surrounding the neck. This is because the active vibration exercise activated the intrafusal receptor inside the muscles and influenced arm and shoulder muscles as well as the muscles around the neck, which increased muscle activity and inhibited muscle activation of unnecessary muscles, thus improving muscle imbalance. ${ }^{16}$ Also, stress of muscles around neck decreased through the dynamic functional improvement of neck and posture-maintaining ability improvement, decreasing the neck disorder index.

After application of active vibration exercise to patients with forward head posture using Flexi-Bar, a significant difference was observed in the muscle activities of the upper trapezius, lower trape- 
zius and serratus muscles. Oliver et al. ${ }^{17}$ reported that application of active vibration exercise had a positive impact on the muscle activity of the shoulder joint, supporting the results of this study. It has been proposed that vibration stimulus can enhance muscular strength and flexibility, and may improve muscular contractions by stimulating muscles physiologically and impacting muscle spindles. ${ }^{18,19}$ The result of electromyogram measurement after the active vibration exercise showed that the muscle activity value of upper trapezius decreased, because muscle tension by the forward head posture was inhibited by the active vibration stimulation. ${ }^{20}$ Active vibration exercise promotes proprioceptor sense by activating the intrafusal shoulder joint and providing strong sense to serratus anterior, increasing muscle activity and contributing to the stability of shoulder joint. ${ }^{20,21}$ Active vibration exercise improves the proprioceptor sense by stimulating the joint capsule around the shoulder joint and the ligaments around the joint. ${ }^{21}$ Yet, these are not only because of the simultaneous contraction and the activation of dynamic stable muscles in the shoulder joint caused by the vibration exercise; while vibration exercises being applied, the mobilization of $a$-exercise nerve cells increases which enhances the ability of nervous muscle adjustment, strengthens large muscles, and increases the proprioceptor feedback, bringing positive impacts to muscle activity. ${ }^{22,23}$

Kasai et al. ${ }^{19}$ stated that the activation of muscle spindle receptors caused by vibration exercise has a positive impact on not only the muscles directly receiving vibration stimulus but also the surrounding muscles. This is considered to be due to the low frequency active vibration stimulus generated by the flexi-bar which is more effective than passively delivered vibration stimulus in improving muscle activity and activating the proprioceptive sense..$^{10,12}$ Active vibration stimulation of low frequency caused by the active vibration exercise is more effective than vibration stimulation delivered passively. ${ }^{24}$ Also, slow vibration stimulation causes tense vibration reflex to belly and tendon, activating proprioceptor sense feedback and thus causing this phenomenon. ${ }^{24,25}$ Also, vibration stimulation provided actively to the shoulder joint influences the stabilization of body and shoulder bone adjustment through exercise chain, causing simultaneous contraction. ${ }^{25,26}$ This leads to sequential muscle activation and influences the head and neck muscles, which also explain the results. ${ }^{26}$ Overall, active vibration exercise has been demonstrated to have positive effects on improving neck pain, the disability index, and muscle activity of patients with forward head posture.
Thus, active vibration exercise is recommended as an effective exercise for patients who have forward head posture to improve their neck pain, functional disorder, and muscle activity. It is also easily accessible and convenient in hospitals and has a possibility to be used at home for continuous exercise.

One key limitation of this study was the short time duration and a lack of follow-up; hence, the long-term effects of the study could not be determined. Moreover, the small sample size is insufficient to generalize the results to all patients with forward head posture. Additional studies addressing these problems is considered necessary.

\section{ACKNOWLEDGEMENTS}

This study was supported in part by research funds from Nambu University, 2018.

\section{REFERENCES}

1. Côté P, Cassidy JD, Carroll L. The saskatchewan health and back pain survey. The prevalence of neck pain and related disability in Saskatchewan adults. Spine. 1998;23(15):1689-98.

2. Kim IG, Lee SY. The effect of forward head posture and tension type headache on neck movement: for office worker. J Kor Phys Ther. 2018; 30(4):108-11

3. Tan LA, Straus DC, Traynelis VC. Cervical interfacet spacers and maintenance of cervical lordosis. J Neurosurg Spine. 2015;22(5):466-9

4. Tepper M, Vollenbroek-Hutten MM, Hermens HJ et al. The effect of an ergonomic computer device on muscle activity of the upper trapezius muscle during typing. Appl Ergon. 2003;34(2):125-30.

5. Decker MJ, Hintermeister RA, Faber KJ et al. Serratus anterior muscle activity during selected rehabilitation exercises. Am J Sports Med. 1999; 27(6):784-91.

6. Masaracchio M, Cleland JA, Hellman M et al. Short-term combined effects of thoracic spine thrust manipulation and cervical spine nonthrust manipulation in individuals with mechanical neck pain: a randomized clinical trial. J Orthop Sports Phys Ther. 2013;43(3):118-27.

7. Torvinen S, Kannus P, Sievänen $\mathrm{H}$ et al. Effect of four-month vertical whole body vibration on performance and balance. Med Sci Sports Exerc. 2002;34(9):1523-8.

8. Lebedev MA, Poliakov AV. Analysis of the interference electromyogram of human soleus muscle after exposure to vibration. Neirofiziologiia. 1991;23(1):57-65.

9. Bosco C, Colli R, Introini E et al. Adaptive responses of human skeletal muscle to vibration exposure. Clin Physiol. 1999;19(2):183-7.

10. Mileva KN, Kadr M, Amin N et al. Acute effects of flexi-bar vs. shambar exercise on muscle electromyography activity and performance. J Strength Cond Res. 2010;24(3):737-48.

11. Lee DK, Kim YN, Park CB et al. The effect of actively induced vibration 
using shoulder joint on pain and dysfunction in patients with low back pain. J Phys Ther Sci. 2018;30(1):23-6.

12. Buteau JL, Eriksrud O, Hasson SM. Rehabilitation of a glenohumeral instability utilizing the body blade. Physiother Theory Pract. 2007;23(6): $333-49$.

13. Lee DK, Kim EK. Effects of active vibration exercise on trunk muscle activity, balance, and activities of daily living in patients with chronic stroke. J Kor Phys Ther. 2018;30(4):146-50.

14. Park CB, Jung EH, Lee HS. A study on body blade training of convergency relation of trunk muscles activity and pain in patients with low back pain. Journal of the Korea Convergence Society. 2017;8(11):12331.

15. Shilpapriya M, Jayanthi M, Reddy VN et al. Effectiveness of new vibration delivery system on pain associated with injection of local anesthesia in children. J Indian Soc Pedod Prev Dent. 2015;33(3):173-6.

16. Kim EK. The effect of body blade training on body alignment of neck and shoulder, muscle activity, stability, and foot pressure at forward head posture. Daegu University. Dissertation of Doctorate Degree. 2015.

17. Oliver GD, Sola M, Dougherty C et al. Quantitative examination of upper and lower extremity muscle activation during common shoulder rehabilitation exercises using the body blade. J Strength Cond Res. 2013; 27(9):2509-17.

18. Issurin VB, Tenenbaum G. Acute and residual effects of vibratory stimu- lation on explosive strength in elite and amateur athletes. J Sports Sci. 1999;17(3):177-82.

19. Kasai T, Kawanishi M, Yahagi S. The effects of wrist muscle vibration on human voluntary elbow flexion-extension movements. Exp Brain Res. 1992;90(1):217-20.

20. Griffin MJ. Hand book of human vibration. 3rd ed. Amsteradam, Elsvier, 2004:171-220.

21. Bogaerts A, Verschueren S, Delecluse C et al. Effects of whole body vibration training on postural control in older individuals: a 1 year randomized controlled trial. Gait Posture. 2007;26(2):309-16.

22. Davies GJ, Dickoff-Hoffman S. Neuromuscular testing and rehabilitation of the shoulder complex. J Orthop Sports Phys Ther. 1993;18(2):449-58.

23. Paine R, Voight ML. The role of the scapula. Int J Sports Phys Ther. 2013; 8(5):617-29.

24. Hodges PW, Richardson CA. Relationship between limb movement speed and associated contraction of the trunk muscles. Ergonomics. 1997;40(11):1220-30.

25. Seidel H. Myoelectric reactions to ultra-low frequency and low-frequency whole body vibration. Eur J Appl Physiol Occup Physiol. 1988;57(5): 558-62.

26. Cools AM, Dewitte V, Lanszweert F et al. Rehabilitation of scapular muscle balance: which exercises to prescribe? Am J Sports Med. 2007; 35(10):1744-51. 